\title{
Factors associated with multiple barriers to access to primary care: an international analysis
}

\author{
L. Corscadden ${ }^{1,2^{*}}$ D, J. F. Levesque ${ }^{3,2}$, V. Lewis ${ }^{4}$, E. Strumpf ${ }^{5}$, M. Breton ${ }^{6}$ and G. Russell ${ }^{7}$
}

\begin{abstract}
Background: Disparities in access to primary care (PC) have been demonstrated within and between health systems. However, few studies have assessed the factors associated with multiple barriers to access occurring along the careseeking process in different healthcare systems.

Methods: In this secondary analysis of the 2016 Commonwealth Fund International Health Policy Survey of Adults, access was represented through participant responses to questions relating to access barriers either before or after reaching the PC practice in 11 countries (Australia, Canada, France, Germany, Norway, the Netherlands, New Zealand, Sweden, Switzerland, the United Kingdom, and United States). The number of respondents in each country ranged from 1000 to 7000 and the response rates ranged from $11 \%$ to $47 \%$. We used multivariable logistic regression models within each of eleven countries to identify disparities in response to the access barriers by age, sex, immigrant status, income and the presence of chronic conditions.
\end{abstract}

Results: Overall, one in five adults (21\%) experienced multiple barriers before reaching PC practices. After reaching care, an average of $16 \%$ of adults had two or more barriers. There was a sixfold difference between nations in the experience of these barriers to access. Vulnerable groups experiencing multiple barriers were relatively consistent across countries. People with lower income were more likely to experience multiple barriers, particularly before reaching primary care practices. Respondents with mental health problems and those born outside the country displayed substantial vulnerability in terms of barriers after reaching care.

Conclusion: A greater understanding of the multiple barriers to access to PC across the stages of the care-seeking process may help to inform planning and performance monitoring of disparities in access. Variation across countries may reveal organisational and system drivers of access, and inform efforts to improve access to PC for vulnerable groups. The cumulative nature of these barriers remains to be assessed.

Keywords: Primary care, Accessibility of healthcare services, Vulnerable groups, Mental health, Healthcare disparities

\section{Background}

Improving access to primary care (PC) is a goal of most healthcare systems. Disparities in access to care have been shown to exist between and within countries [1-5]. These disparities in access to PC in turn contribute to disparities in health, while improving access for vulnerable groups helps to reduce gaps in health outcomes [6,7].

\footnotetext{
* Correspondence: lisa.corscadden@health.nsw.gov.au

${ }^{1}$ Australian Institute of Tropical Health and Medicine, James Cook University,

Townsville, QLD 4812, Australia

${ }^{2}$ Bureau of Health Information, Level 11, 67 Albert Avenue, Chatswood, NSW

2067, Australia

Full list of author information is available at the end of the article
}

There are various ways to conceptualise access. From a patient perspective, access to care has been conceptualized as a process from perceiving a need for care and seeking care, to reaching and obtaining care and benefiting from the services received [8]. Reasons for unmet needs for healthcare have been demonstrated to exist at many stages, including in areas of availability, affordability and acceptability both before and after physically reaching a provider $[9,10]$.

From an empirical perspective, disparities in access to PC have been documented across a range of measures and vulnerable groups, with some consistencies and 
areas of divergence across countries. Foregoing care due to cost, difficulties with after-hours primary care, and timely access to PC appointments are more commonly experienced by people in lower income groups $[5,11-$ 13]. Racial minorities and immigrants have lower rates of affiliation with a regular care provider and more unmet health needs, with disparities more pronounced in the United States than in Canada [14]. In many countries, people with multiple chronic conditions are more likely to have difficulties accessing after-hours care, and report having to wait several days to get an appointment when sick, compared to people with no conditions [5]. People with chronic conditions, particularly people with mental health conditions, were found to be more likely to forego care due to cost and have higher out-of-pocket healthcare costs than people with no chronic conditions [15].

When barriers to access to care accumulate, there are impacts upon healthcare use patterns. For example, studies have found a higher number of barriers to access were associated with more intensive use of emergency care in general [16] and for primary care reasons [17]. There is limited research investigating disparities in multiple barriers to access to PC and how patterns differ across countries. Therefore, this secondary data of an international survey systematically examines which population groups were more likely to experience multiple barriers to accessing PC across a range of measures, population groups and countries.

\section{Methods}

We used the 2016 Commonwealth Fund International Health Policy Survey of adults aged 18 years and over in 11 countries: Australia, Canada, Germany, France, Netherlands, New Zealand, Norway, Sweden, Switzerland, the United Kingdom and the United States. Analyses were weighted so that the estimates were representative of the age, sex, regional and education profile of adults in each country. The number of respondents ranged between countries from 1000 to 7000 adults (Additional file 1: Appendix 1).

Our choice of access measures in this analysis was based on the conceptual model proposed by Levesque et al. [8] and followed an iterative prioritising process based on local innovation partnerships input described elsewhere [18] with additional considerations from key literature [17]. Responses were dichotomised (where applicable, responses of 'sometimes, rarely and never' were categorised as no, and 'always and often' grouped as yes). Next, measures were grouped into barriers experienced: before reaching a PC provider (no regular care provider; difficulties in accessing after-hours; difficulties in getting timely appointment or response to call; skipping tests, medication, or care due to cost) and after reaching a PC provider (regular care provider did not: listen carefully; know medical history; coordinate care, or spend enough time). The number of barriers experienced before and after reaching care for each person was calculated.

We considered vulnerable groups who may be more likely to face barriers to access to care as being those participants with chronic conditions, lower income, females, people over 65 , and those not born in the country where they reside. In terms of chronic conditions, respondents were categorised into three groups: 1) people with no conditions; 2) those who said they had been diagnosed with a physical condition (including joint pain or arthritis, asthma or chronic lung disease, cancer, diabetes, heart disease, hypertension, high blood pressure, or stroke); and 3) those who said they were told by a doctor they had a mental health condition (regardless of the fact that they may have had other physical conditions).

For each country, the percentage of each vulnerable group, access barrier and combination of access barriers by group was calculated as well as a country average. Multivariable logistic regression models were run using SAS/STAT software, Version 9.3 (Copyright @ 2005 SAS Institute Inc.) for each of the 11 countries to assess the likelihood of experiencing barriers to access to PC, adjusting for age, sex, income, chronic conditions, immigrant status as well as hospitalisation in the previous two years as a proxy for more intensive health service use. Our primary outcomes of interest are experiencing multiple barriers: 1) before; and 2) after reaching PC. As secondary outcomes, we considered each access barrier individually, calculating the number of times each vulnerable group was significantly more likely to experience barriers than a corresponding reference group was calculated across all countries (significant adjusted odds ratio where $p<0.05)$. The number of times each population group was significantly more likely to face barriers for each country was also summarised.

\section{Results}

One fifth of adults (21\%) on average reported experiencing two or more barriers before reaching PC (ranging from $6 \%$ to $38 \%$ across countries). After reaching PC, among adults who had a regular care provider, $16 \%$ reported experiencing two or more barriers (ranging from $5 \%$ to $30 \%$ across countries) (Table 1 ).

Figure 1 shows the average percentage of people experiencing multiple barriers by vulnerable group. People with mental health conditions, those below average income, and those born outside their country of residence were more likely to face multiple barriers, whereas seniors were less likely to experience any barriers to access PC. Country level results for these four vulnerable 


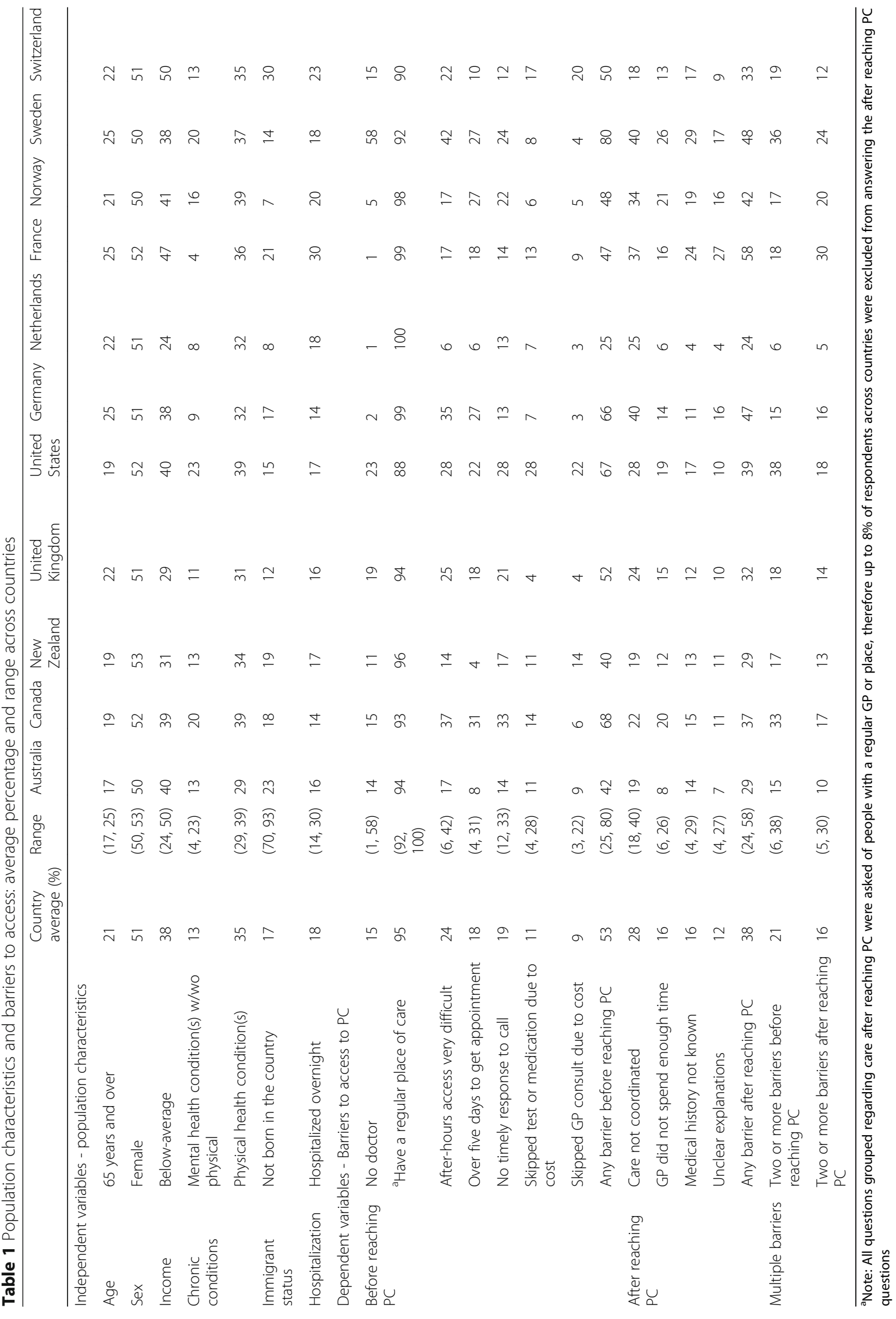




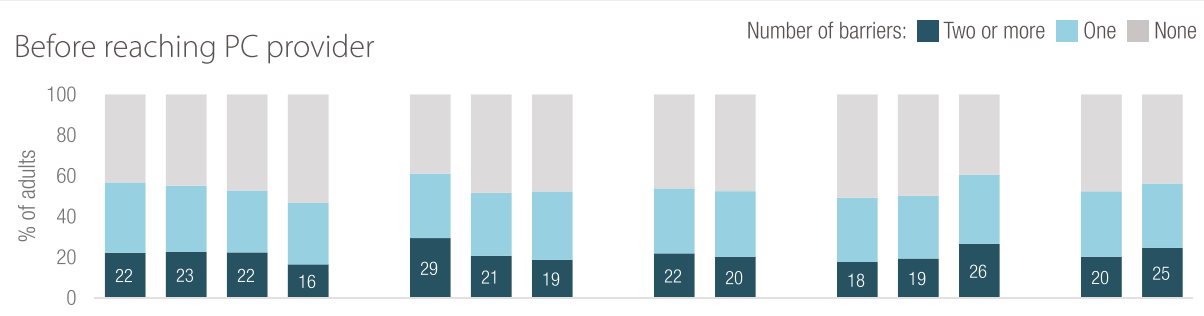

\section{After reaching PC provider}

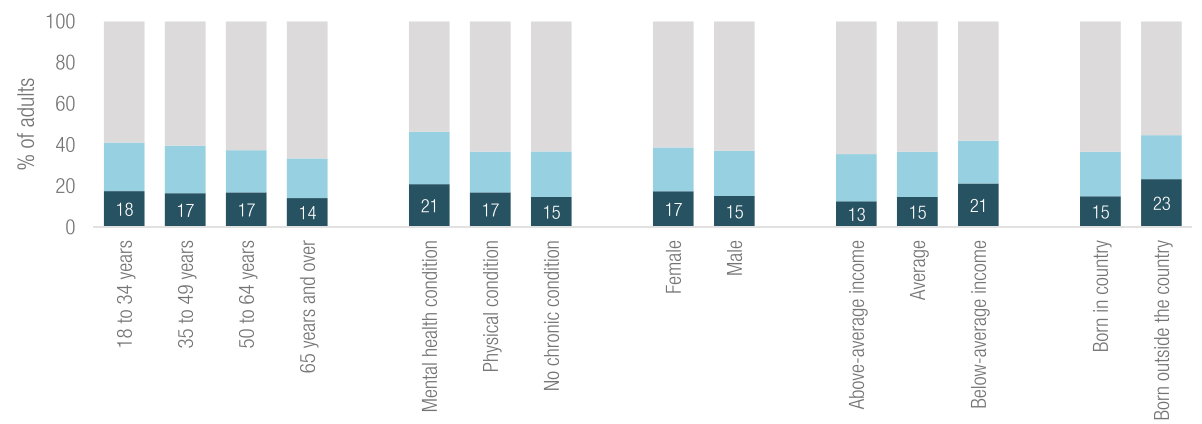

Fig. 1 Percentage of adults experiencing multiple barriers to access both before and after reaching PC, average of countries by population characteristics. Note: Descriptive results based on unadjusted country averages, full results available in the technical appendix

groups are shown in Fig. 2. Before reaching PC, people with mental health conditions were more likely to experience multiple barriers, particularly in Australia and NZ. After reaching PC, people who were not born in the country appeared more likely to experience barriers, particularly in Norway, France, the UK, Switzerland, and the US.

Results of multivariable models estimating the odds of experiencing multiple (two or more) barriers before and after reaching care are summarised in Fig. 3 (full results are provided in the technical appendix showing country specific values and significance of each result). In most countries, people aged 65 years and over are less likely to experience multiple barriers than younger adults. People with below-average income were more likely to experience multiple barriers after reaching care with adjusted odds ratios (AOR) greater than one for all countries. Before reaching $\mathrm{PC}$, people with below-average income, physical health conditions, and mental health conditions were more likely to experience barriers in all but one country (the UK, France and NZ for each of the vulnerabilities respectively). Being born outside the country of residence was associated with multiple barriers particularly after reaching $\mathrm{PC}$ for the US, Switzerland, France, Canada and Australia (AOR ranged from 1.59 to $3.12, p<0.05$ ). There were few significant differences by sex, however females were more likely to experience multiple barriers: before reaching care in NZ (AOR $1.99 \mathrm{p}<0.05$ ), and after reaching care in France and Sweden (AOR. 1.49 and 1.39 respectively, the AOR for NZ was higher but not significant).
The adjusted odds ratios for each access barrier individually as well as multiple barriers are provided in Fig. 4, to determine whether findings for multiple barriers are consistent across access measures. For almost all access measures older age was protective and below-average income was associated with a greater likelihood of barriers. However, in some countries older people experienced more barriers with timely access to GP care and receiving clear explanations.

For people with mental health conditions the extent of barriers in access to PC varied by country and type of access barrier. People with mental health conditions were consistently more likely to face affordability barriers; foregoing consultations and medication and tests due to cost across countries (AOR ranged from 1.20 to 4.83). However, they were less likely to say they had no regular care provider, or have long waits to see a GP compared to people with no mental health conditions in some countries.

A summary of the number of times each population group was significantly more likely to face barriers is presented in Fig. 5. For each country and access measure combination, we ran a model to estimate the odds of experiencing barriers for all vulnerable groups considered. In total, 106 models were generated where there were sufficient respondents. The most common difference was for people with below-average income, who were significantly more likely to experience access barriers than the above-average income group in 50 of 106 possible models. People with a mental health condition were more likely to experience barriers compared to people 


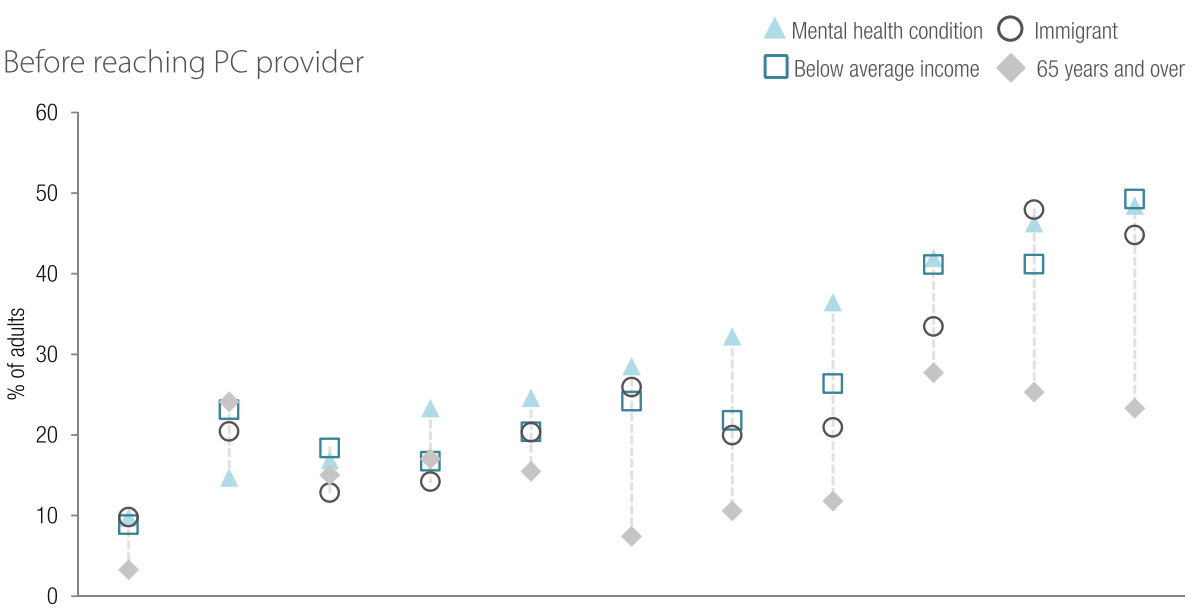

After reaching PC provider

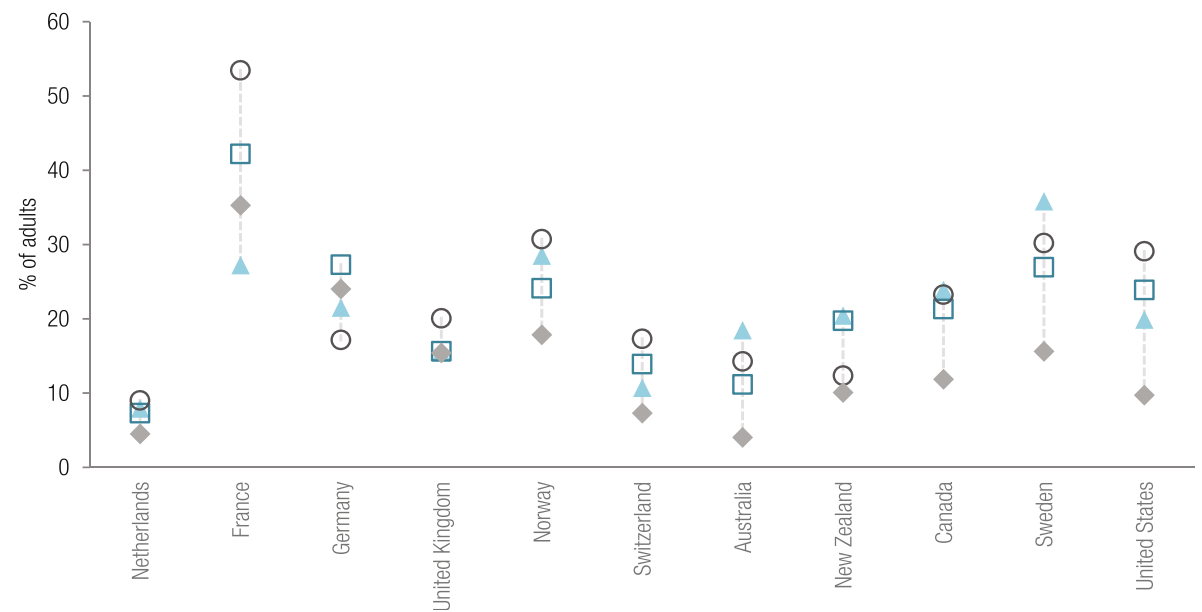

Fig. 2 Percentage of adults reporting experience of multiple barriers to access both before and after reaching PC, by country and four selected vulnerable groups. Note: Descriptive results based on weighted prevalence within countries by population group, sorted by the percentage of people with a mental health condition experiencing barriers before reaching PC. For variables with more than two categories, the group with the most pronounced barriers was selected

with no conditions in 35 models, and people born outside the country they reside in were more likely to experience barriers in 30 models. Older people were less likely than younger people to experience barriers in 45 of 106 models (see the technical appendix for complete results).

\section{Discussion}

Access to PC spans from the identifiction of a health problem, to seeking care, to obtaining an appointment and reaching $\mathrm{PC}$, and is affected by the characteristics of the interaction with the PC provider $[8,10]$. Barriers to access to PC have been shown to vary by country and the measure of access [18]. This study goes further to assess which population groups are more likely to experience multiple access barriers, and summarise how patterns vary by population and measure of access.
Results presented in this secondary analysis of an international survey shows many people experience multiple barriers to care at different points in the pathway of accessing care. Further, certain population groups are disproportionately more likely to experience multiple barriers.

Factors associated with experiencing multiple barriers to access to care were generally consistent across countries. These factors included; below-average income, immigrant status, and chronic conditions, particularly mental health conditions. People with below-average income were more likely to experience barriers after reaching $\mathrm{PC}$ consistently across countries (AOR range across countries: 1.22 to 3.32). People with mental health conditions were more likely than people with no chronic conditions to experience multiple barriers before reaching PC, and statistically significant difference in 6 of 11 


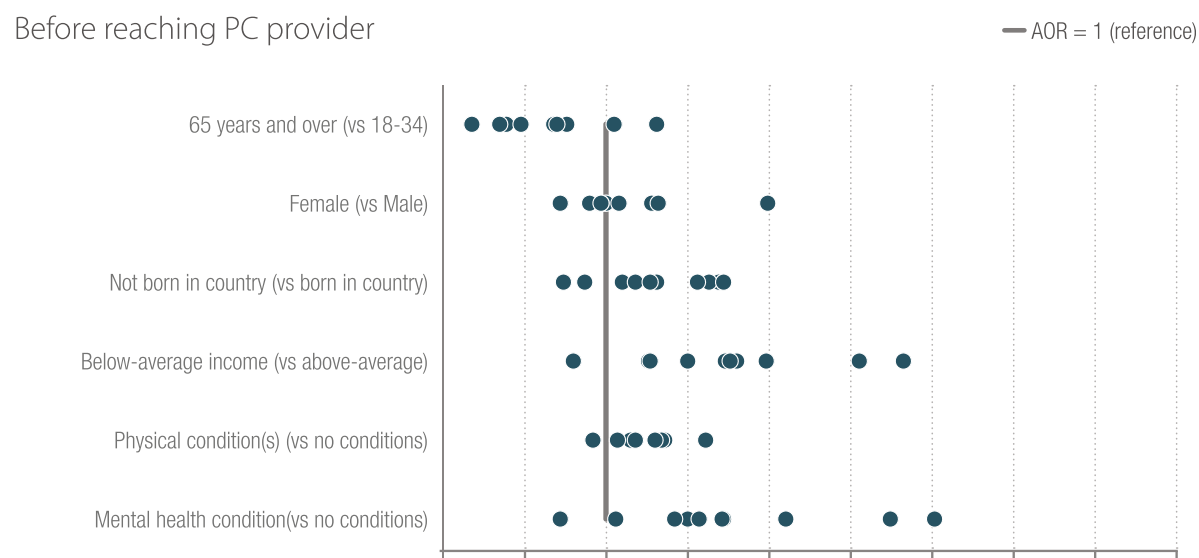

After reaching PC provider

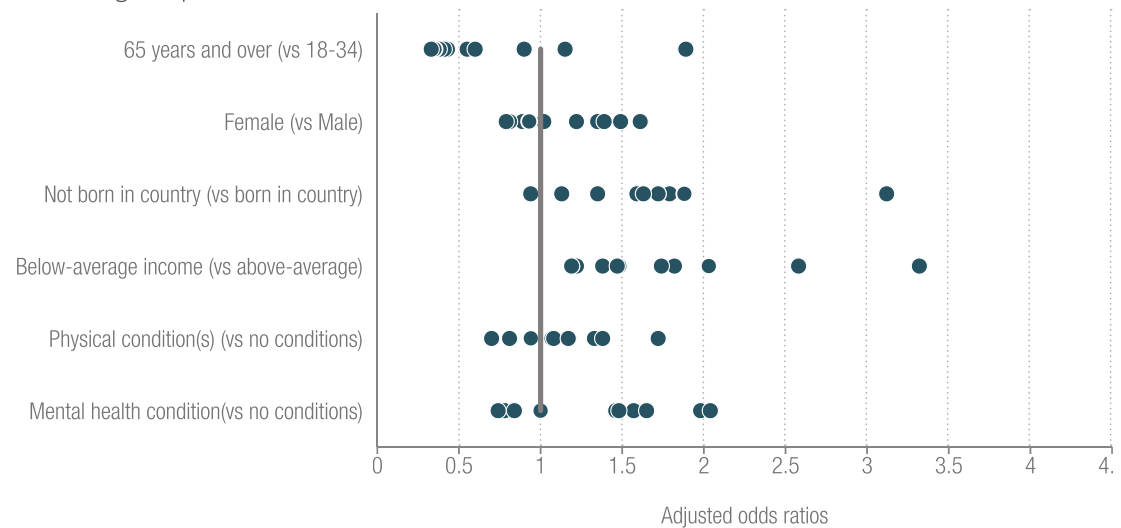

Fig. 3 Adjusted odds ratios of multiple (two or more) barriers to access before and after reaching care across countries and population characteristics. Figure note: The lines represent the $A O R=1$, adjusting for age, sex, immigrant status, income, chronic conditions, and being hospitalised. Each circle represents a country's Adjusted Odds Ratio (AOR) from a full model estimating the outcomes of having multiple barriers to access before and after reaching PC. See the technical appendix for full results by country and access measure. Results are excluded if the country had fewer than 100 respondents with the selected barrier

countries. Immigrants were more likely to experience multiple barriers, pariticuarly after reaching care (AOR ranged from 0.94 to 3.12 ). In contrast, age was protective, with people aged 65 and over less likely to experience barriers in most countries.

Findings regarding income and age were consistent with the literature. Systematic reporting of disparities across 21 measures of access in the United States showed lower income was a risk factor for all measures, with disparities by ethnicity also prevalent but less pronounced [1]. Similarly, we found the number of significant differences by income to be the most persistent disparity across countries. Findings from past Commonwealth Fund International Health Policy surveys have shown that low income was a signficant risk factor across most countries and older age was protective for most access measures [5].

There is broad interest in addressing barriers in access to care for a range of vulnerable groups $[19,20]$. There are many possible insights into why barriers exist or how to address them. In terms of barriers faced by people with low income, qualitative research has suggested provider lack of understanding of living in poverty may lead to the development of inappropriate care plans that do not acknowledge and account for patients' social circumstances [21]. Disparities in access to PC faced by people born outside the country they reside in may indicate issues around seeking care; however, results in this analysis suggested they also experienced disparities after reaching PC, which may reflect language and cultural differences between patients and providers. Finally, for people with mental health conditions, there may be additional factors at play such as the stigmatisation related to seeking care [22] as well as a lack of preparation of PC providers to deal with mental health issues [23].

As access is conceptualised from both provider and patient perspectives in the Levesque et al. model [8], we also consider factors related to both supply of, and demand for care to contribute to the reasons some groups 


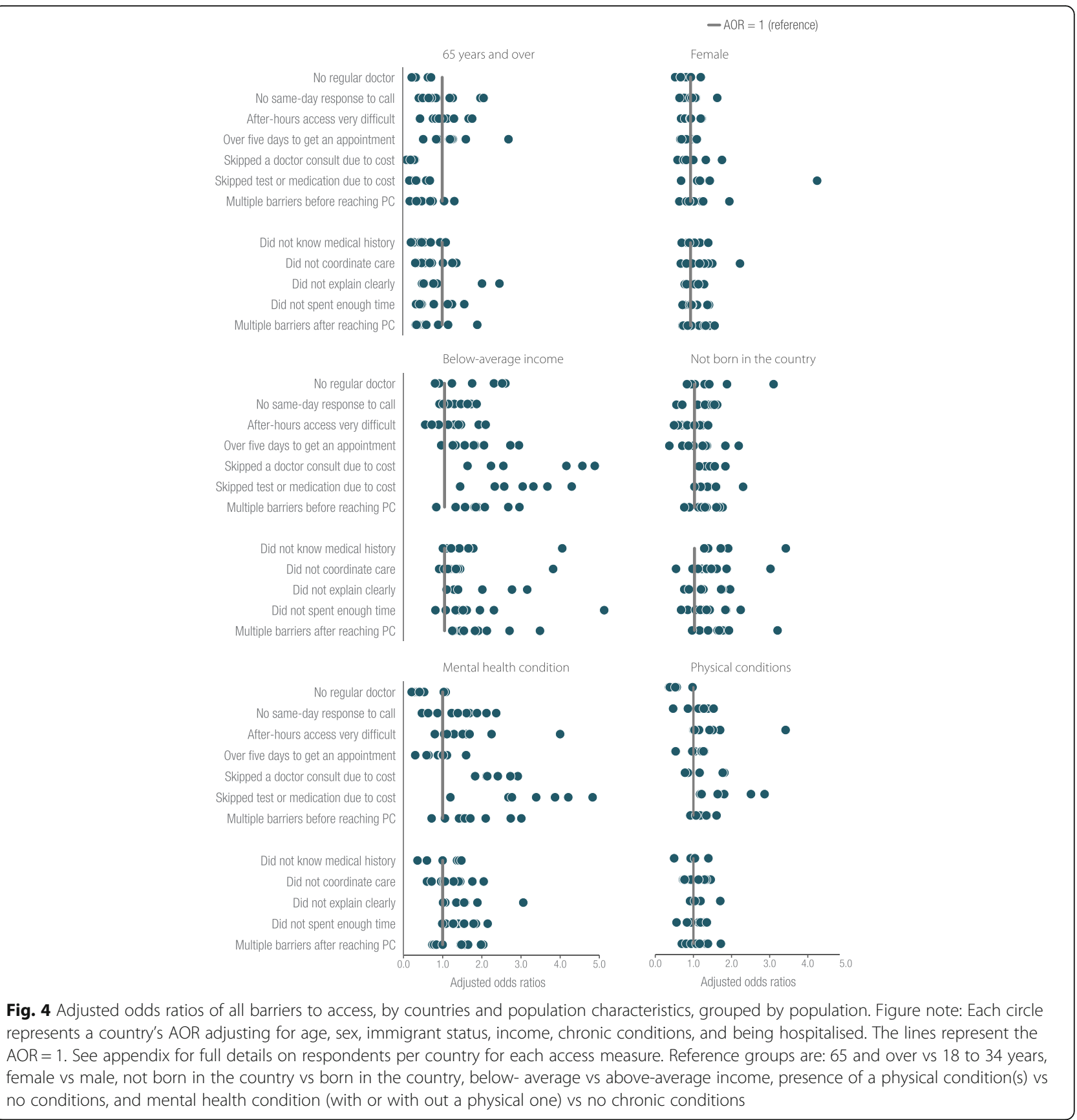

experience multiple barriers to accessing care. In terms of demand, it has been suggested that people with mental health concerns are less likely to seek care, and interventions building trust in their physicians was a protective factor in care-seeking [24]. Provider preference or comfort in managing certain health conditions, particularly mental health, may also be a factor contributing to disparities some groups face. In a regular survey of primary care providers, GPs were asked if they felt their clinic was prepared to manage care for different population groups. Fewer than half of GPs in the 11 surveyed countries said they were well prepared to manage people with serious mental health conditions or substance use issues - consistently lower than perceptions of preparedness to manage multiple chronic conditions [23].

This study suggests that factors associated with barriers to access do not occur in isolation and may be clustered and multifactorial. Research considering multiple risk factors also suggests that clinical and social factors can also accumulate and interact to influence access to care [25]. This model recognises that factors associated 
Worse than reference $\quad$ Not statistically different $\mathbf{B}$ Better than reference

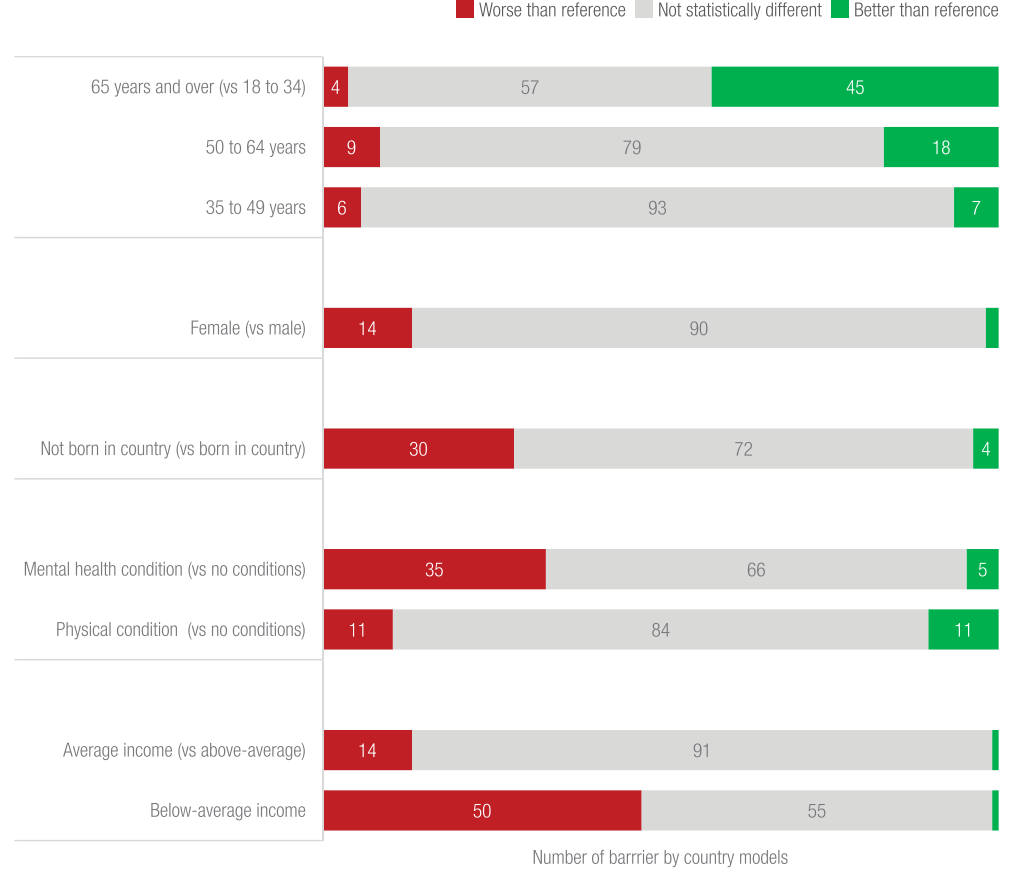

Fig. 5 Number of significant differences for each population compared to reference group across countries and all barriers to access. Figure Note: There were 106 total comparisons where there were sufficient numbers of respondents out of a possible 132 models (11 countries * 12 access measures). Numbers of responses of 'events' or barriers to access for each access measure and country, and results by country are provided in the technical appendix. Results are based on full models adjusting for age, sex,immigrant status, income, chronic conditions, and being hospitalised in the past two years

with poorer access to PC are interconnected, such that people experiencing multiple vulnerability factors may have even greater barriers to PC. For example, developing mental health conditions can impact income, and having lower income can impact mental health [26]. Another model of vulnerability suggests a risk factor profile approach to understanding disparities in healthcare [27]. Research has shown that an increased number of risk factors is associated with a greater likelihood of unmet needs [28]. Future work could consider the possible cumulative effects of multiple factors on the experiences of barriers to access to PC.

\section{Limitations}

There are limitations to this study that should be acknowledged. As it was a secondary analysis, the survey questions did not completely cover all five domains of the conceptual framework of access to care $[8,10]$; therefore, we have considered a simplification of barriers before and after reaching PC. We have assumed the selected measures apply to primary care, rather than all healthcare. The survey also did not include information regarding ethnicity or language spoken for all countries. Futher, rurality and education measures could not be created to be comparable across countries. These population factors are also known to be associated with barriers to access to PC. Finally, the prevalence of people reporting they had mental health problems, ranged from $4 \%$ to $23 \%$ across countries and is likely to be underreported in some countries and through self-reported single item questions. For example, for Australia the prevalence of self-reported mental health problems was $13 \%$, slightly lower than the $17.5 \%$ estimate from the $\mathrm{Na}$ tional Health Survey [29].

There are data limitaitons associated with the survey that affect the comparability of disparity results across countries. First, there were different numbers of respondents and response rates for each country, therefore different power to detect significant differences. Our results reflect patterns across countries in the relationships and do not compare the size of disparities. We address issues of multiple comparisons in a descriptive manner by placing counts of significant differences, alongside descriptive bivariate patterns, as well as the size of odds ratios from multivariable models to identify consistant patterns in findings. Further, as analysis is based on survey data with wide margins of error $(+/-3-$ $4 \%$ ) [4] adjusted odds of 1.5 or more were generally significant, a magnitude which also appears meaningful from a face-validity perspective. Future work to understand the associations between population factors and access barriers over time in each country, as well as 
interactions between factors such as income and gender or health conditions may provide a fuller picture of patterns of disparities.

\section{Conclusions}

There are many differences in the political and economic climates of the countries whose data were analysed; however, our findings show many consistent patterns in disparities of access to PC for various vulnerable groups, as well as some that are more pronounced in certain contexts. Further our findings demonstrate the cumulative nature of barriers preventing people from fully accessing PC. Considering the characteristics of population groups that are more likely to experience barriers to access and the reasons they might have issues seeking, reaching or fully accessing care may help reorient health services to address disparities in access to PC. Country and population group differences in disparities suggest inequities in access are amenable to a range of policy, organisational and educational responses to reduce them.

\section{Additional file}

Additional file 1: Factors associated with multiple barriers to access to primary care - Technical Appendix. (DOCX $86.9 \mathrm{~kb}$ )

\section{Abbreviations \\ AOR: Adjusted Odds Ratio; GP: General Practitioner; IMPACT: Innovative Models Promoting Access to Care Transformation; NZ: New Zealand; PC: Primary care; UK: United Kingdom; US: United States}

\section{Acknowledgements}

Data analyses were conducted as part of The Innovative Models Promoting Access to Care Transformation (IMPACT) Centre for Research Excellence funded by the Australian Primary Health Care Research Institute (APHCRI) and the Canadian Institutes for Health Research (CIHR). The research group works with consumers, policy makers and providers through local innovation partnerships in Australia and Canada to identify organisational innovations designed to improve access to appropriate PHC for vulnerable populations, and establish the effectiveness and scalability of the most promising innovations. The information and opinions contained in this paper do not necessarily reflect the views or policy of these funding agencies.

We also acknolwedege contributions Huei-Yang Chen for discussions on SAS coding, Ed Bury in preparing images for submission, as well as other IMPACT team members including Riki Lane in the discussion of this work as part of project team meetings.

\section{Concent for pubication}

Not applicable.

\section{Funding}

This study has been conducted as part of IMPACT (Improving Models Promoting Access-to-Care Transformation Program). IMPACT is funded by the CIHR Signature Initiative in Community-Based Primary Health Care, the Fonds de recherche du Québec - Santé, and the Australian Primary Health Care Research Institute (which is supported by a grant from the Australian Government Department of Health.

Work by LC and JFL is supported by the Bureau of Health Information in New South Wales.

ES and MB are supported by a chercheur boursier Junior 2 from the Fonds de Recherche du Québec - Santé.
Availability of data and materials

Commonwealth fund survey website: http://www.commonwealthfund.org/ interactives-and-data/international-survey-data.

\section{Authors' contributions}

LC completed data analysis with support from ES, JFL. LC drafted the manuscript for publication and JFL, ES, VL contributed to the content and revision of the manuscript. GR contributed to working group meetings and revisions. MB contributed to revisions. LC managed revisions, literature and checking of the manuscript. All authors read and approved the final version.

Ethics approval and consent to participate

Not applicable.

\section{Competing interests}

The authors declare that they have no competing interests.

\section{Publisher's Note}

Springer Nature remains neutral with regard to jurisdictional claims in published maps and institutional affiliations.

\section{Author details}

${ }^{1}$ Australian Institute of Tropical Health and Medicine, James Cook University, Townsville, QLD 4812, Australia. ${ }^{2}$ Bureau of Health Information, Level 11, 67 Albert Avenue, Chatswood, NSW 2067, Australia. ${ }^{3}$ Centre for Primary Health Care and Equity, University of New South Wales, Sydney, NSW 2052, Australia. ${ }^{4}$ Australian Institute for Primary Care \& Ageing, La Trobe University, Melbourne, VIC 3068, Australia. ${ }^{5}$ Department of Economics and Department of Epidemiology, Biostatistics, and Occupational Health, McGill University, 855 Sherbrooke St. West, Montreal, QC H3A 2T7, Canada. ${ }^{6}$ Department of community health, University of Sherbrooke, 150 Place Charles LeMoyne, Longueil, Québec J4K OA8, Canada. ${ }^{7}$ General Practice Research, School of Primary and Allied Health Care, Monash University, 270 Ferntree Gull Rd Notting Hill, Melbourne, VIC 3168, Australia.

Received: 2 July 2017 Accepted: 6 February 2018

Published online: 20 February 2018

\section{References}

1. Agency for Healthcare Research and Quality. National Healthcare Quality and Disparities Report: Chartbook on Access to Health Care 2015: Agency for Healthcare Research and Quality, Rockville; 2014.

2. Australian Institute for Health and Welfare. Australia's health 2016. Canberra: AlHW; 2016

3. OECD. Health at a Glance 2015: OECD Indicators. Paris: O. Publishing; 2015.

4. Schoen C, et al. Access, affordability, and insurance complexity are often worse in the United States compared to ten other countries. Health Aff. 2013;32(12):2205-15

5. Schoen $C$, et al. How health insurance design affects access to care and costs, by income, in eleven countries. Health Aff. 2010;29(12):2323-34.

6. Shi L. The impact of primary care: a focused review. Scientifica. 2012;2012:22.

7. Starfield B, Shi LY, Macinko J. Contribution of primary care to health systems and health. Milbank Q. 2005;83(3):457-502.

8. Levesque JF, Harris MF, Russell G. Patient-centred access to health care: conceptualising access at the interface of health systems and populations. Int J Equity Health. 2013;12:12-8.

9. Sibley LM, Glazier RH. Reasons for self-reported unmet healthcare needs in Canada: a population-based provincial comparison. Healthcare Policy. 2009; 5(1):87-101

10. Ward B, et al. Which dimensions of access are most important when rural residents decide to visit a general practitioner for non-emergency care? Aust Health Rev. 2015;39(2):121-6.

11. Davis K, Ballreich J. Equitable access to care-how the United States ranks internationally. N Engl J Med. 2014;371(17):1567-70.

12. De Looper M, Lafortune G. Measuring disparities in health status and in access and use of health care in OECD countries. OECD Health Working Papers. 2009:(43):1.

13. Osborn R, et al. In new survey of eleven countries, US adults still struggle with access to and affordability of health care. Health affairs (Project Hope). 2016;35(12):2327. 
14. Lasser KE, Himmelstein DU, Woolhandler S. Access to care, health status, and health disparities in the United States and Canada: results of a crossnational population-based survey. Am J Public Health. 2006;96(7):1300-7.

15. Callander EJ, Corscadden L, Levesque J-F. Out-of-pocket healthcare expenditure and chronic disease - do Australians forgo care because of the cost? Aust J Prim Health. 2016;23:15-22.

16. Cheung PT, et al. National study of barriers to timely primary care and emergency department utilization among Medicaid beneficiaries. Ann Emerg Med. 2012;60(1):4-10. e2

17. Macinko J, et al. Gaps in primary care and health system performance in six Latin American and Caribbean countries. Health Aff. 2016;35(8):1513-21.

18. Corscadden L, et al. Barriers to accessing primary health care: comparing Australian experiences internationally. Aust J Primary Health. 2017;23(3):223-8.

19. Richard $L$, et al. Equity of access to primary healthcare for vulnerable populations: the IMPACT international online survey of innovations. Int J Equity Health. 2016;15(1):64.

20. Grabovschi C, Loignon C, Fortin M. Mapping the concept of vulnerability related to health care disparities: a scoping review. BMC Health Serv Res. 2013;13(1):94

21. Bloch G, Rozmovits L, Giambrone B. Barriers to primary care responsiveness to poverty as a risk factor for health. BMC Fam Pract. 2011;12(1):62.

22. Thornicroft G. Stigma and discrimination limit access to mental health care. Epidemiol Psichiatria Sociale. 2008;17(01):14-9.

23. Bureau of Health Information. Healthcare in Focus 2015. How does NSW compare. Sydney: BHI; 2016.

24. Thompson $A E_{\text {, et }}$ al. The influence of gender and other patient characteristics on health care-seeking behaviour: a QUALICOPC study. BMC Fam Pract. 2016;17(1):38.

25. Shippee ND, et al. Cumulative complexity: a functional, patient-centered model of patient complexity can improve research and practice. J Clin Epidemiol. 2012;65(10):1041-51.

26. Schofield DJ, et al. The personal and national costs of mental health conditions: impacts on income, taxes, government support payments due to lost labour force participation. Bmc Psychiatry. 2011;11:72.

27. Shi $L$, et al. Enhancing the measurement of health disparities for vulnerable populations. J Public Health Manag Pract. 2008;14(6):S45-52.

28. Shi LY, Stevens GD. Vulnerability and unmet health care needs - the influence of multiple risk factors. J Gen Intern Med. 2005;20(2):148-54.

29. Australian Bureau of Statistics. National Health Survey: First Results, 2014-15. Cat. no. 4364.0.55.001 [Online]. ABS. 2015. http://www.abs.gov.au/AUSSTATS/ abs@.nsf/DetailsPage/4364.0.55.0012014-15?OpenDocument\#Publications.

\section{Submit your next manuscript to BioMed Central and we will help you at every step:}

- We accept pre-submission inquiries

- Our selector tool helps you to find the most relevant journal

- We provide round the clock customer support

- Convenient online submission

- Thorough peer review

- Inclusion in PubMed and all major indexing services

- Maximum visibility for your research

Submit your manuscript at www.biomedcentral.com/submit 